\title{
Bipolar Disorder and Wilson's Disease: A Case Report
}

\author{
Bhagat $\mathrm{S}^{1}$, Nepal H${ }^{2}$, Verma $\mathrm{AK}^{3}$
}

1. Clinical observer, St. Mary's Hospital, Decatur, IL, USA 2. MD Resident Physician, Department of Psychiatry, Southern Illinois University, Springfield, IL, USA 3. Assistant Professor in Department of Psychiatry, Janaki Medical College, Janakpur, Nepal

E-mail *Corresponding author: shambhu_bhagat@yahoo.com

\begin{abstract}
Wilson's disease is an uncommon inherited disorder characterized by low serum ceruloplasmin levels, hypercupriuria and Kayser-Fleischer rings. Here we describe the case of a young boy who presented with symptoms of bipolar depression along with bilateral hand tremors. He was started with Quetiapine but symptoms did not improve. Investigations revealed cirrhotic changes of liver, low serum ceruloplasmin levels, presence of Kayser-Fleischer rings. The diagnosis of Wilson's disease was confirmed by high 24hr. urinary copper levels. Bipolar symptoms improved after 7 months of initiation of oral penicillamine treatment.
\end{abstract}

Key words: Bipolar disorder; Ceruloplasmin; Wilson's disease

\section{INTRODUCTION}

Wilson's disease (WD) is an inherited autosomal recessive disorder that affects copper metabolism and is caused by mutation in a gene on chromosome 13 that encodes ATP 7B. ${ }^{1}$ Different psychiatric manifestations of WD have been reported frequently as schizophrenia-like psychosis, cognitive impairment, dementia, anxiety, and behavior abnormalities and personality disorders. ${ }^{2}$ Depression is often seen among patient with WD affecting approximately $30-60 \%, 3$ however hypomania, frank mania and bipolar affective disorder (BPAD) are rarely found in clinical practice. 4 The purpose of this case study is to determine the bipolar affective symptoms associated with WD. Since very few similar case is seen in clinical practice, this case report may help us in understanding and diagnosing such type of cases when encountered in our clinical practice.

\section{CASE REPORT}

A 21 year old gentleman presented to the clinic (Janaki Medical College) with features consistent with depression. Patient has a history of depressed mood, decrease energy and appetite with decrease interest and recurrent suicidal thoughts without specific plan since 3 weeks. Mental status examination revealed depressed mood with restricted affect, decreased psychomotor activity, impaired attention and concentration. Neurological examination finding was normal except for coarse bilateral upper extremities tremor.

Detailed collateral history revealed a manic episode 17 months back for which patient was treated with Valproate (in India). Patient developed tremors in both hand 5 months after initiation of treatment. When patient was seen during the follow up visit, it was explained as a side effect of Valproate. He was thus started on Propranolol. Despite being on Propranolol, tremors continued to progress in severity. With little relief from Propranolol, patient discontinued its use on his own. There was no known medical, surgical or substance abuse history.

Patient was advised for admission for further workup and evaluation. He refused to do so. Patient was started with Quetiapine and suicide precaution was explained to patient and his father. During the 2 months follow up, symptoms persisted. Moreover, his bilateral hands tremor was progressive. Patient was thoroughly investigated. Liver function test 
was found deranged and the USG abdomen revealed early cirrhotic changes. Considering the investigation findings, WD was suspected for which ophthalmology consultation was done and Kayser-Fleischer(KF) rings was detected on slit lamp examination. After suspicion of Wilson's disease, Quetiapine was stopped.

Due to limited access, ceruloplasmin test could not be done. Patient was then referred to higher centre (in India). Further work-up revealed decreased ceruloplasmin levels (16.4 $\mathrm{mg} \%$ ) and an increased $24 \mathrm{hr}$ urinary copper levels $(10,900 \mu \mathrm{g} / \mathrm{d})$. However liver biopsy was not done. A provisional diagnosis of WD was made. Patient was then started on oral penicillamine. On follow up with us at 7 months of initiation of treatment, patient was found to be completely asymptomatic.

\section{DISCUSSION}

WD is an autosomal recessive disorder of copper metabolism where there is toxic accumulation of copper in liver, brain and eye and is known as hepatolenticular degeneration. ${ }^{1}$ It is found to be associated with several psychiatric disorders however Bipolar Affective Disorder is a rare presentation in WD. ${ }^{4}$

A study showed an association between BPAD and WD using standardized diagnostic tools and a case control design. ${ }^{5}$ Similarly another study found out the higher association between bipolar disorder and WD among 119 patients with neurological manifestations. ${ }^{6}$ The study further pointed out a patient with WD whose initial manifestation was a manic episode followed by depression whose presentation was consistent with our study. Another case of mania as the first manifestation of WD was described by Kumar et $\mathrm{al}^{7}$ in a 18 year old boy who presented to a psychiatric clinic with manic features and extra-pyramidal symptoms. This case pointed out the need for detailed evaluation during such presentation to rule out underlying neuropsychiatric conditions.

For the first time when patient complains of tremor of both hands, this misleads the physician as the side effect of Valproate and he was started on Propanolol. Although tremor is commonly seen in clinical practice among patients on mood stabilizers, other differential diagnosis of neurological conditions needs to be considered. When patient presented to our department, we made the provisional diagnosis of bipolar depression after detailed history and examination, thus Quetiapine was started. Since informant refused further investigation, Quetiapine was initiated based on the past evidence. ${ }^{8}$ Majority of patients and informants refuse undertaking investigation prior to initiation of treatment in our setting although it is suggested to all. The initiation of early work-up would have accelerated for an early diagnosis and appropriate treatment in our case.

As bipolar symptoms were persistent along with tremor of hand, we had advised for some relevant investigations that revealed cirrhosis of liver, KF ring on slit lamp examination, and low ceruloplasmin level along with increased $24 \mathrm{hr}$ urinary copper levels. These clinical and investigation findings were consistent with WD and also supported by past studies. ${ }^{1,6}$ After investigation results, we made strong suspiciousness of WD and Quetiapine was discontinued. Past studies revealed that there is no need of treatment of bipolar symptoms to young individuals having WD.,4 These studies further suggest that treatment of WD alone improve the depressive or manic symptoms in bipolar disorder. Patient was asymptomatic after 7 months of initiation of penicillamine. Other studies also revealed the improvement of bipolar symptoms in patients with WD but the duration of response differ in individual. $4,5,7$

\section{CONCLUSION}

WD should be suspected in any young patient who presents with psychiatric symptoms along with neurological problems. Further studies are necessary for the evaluation of BPAD in Wilson's disease among young individuals. All relevant investigations must be advised prior to initiation of treatment to rule out organic pathology and to diagnose and initiate treatment at the earliest. This largely decreases the morbidity and mortality associated with such conditions. 


\section{REFERENCE}

1.Trocello JM: Wilson France: a national database for Wilson's disease. Orphanet J RareDis. 2010;5(1):21.

2. Akil M, Brewer GJ. Psychiatric and behavioral abnormalities in Wilson's disease. Adv Neurol. 1995;65:171-78.

3. Hesse S, Barthel H, Hermann W, Murai T, Kluge $\mathrm{R}$, Wagner A, et al. Regional serotonin transporter availability and depression are correlated in Wilson's disease. J Neural Transm. 2003;110(8):92333.

4. Costa MA, Mitiko DM, Caixeta L, Spitz M, Tavares LL, Reis BE. Mania first manifestation Wilson's disease Bipolar Disorders. 2008;10(3):44750.

5. Carta MG, Sorbello O, Moro MF, Bhat KM, Demelia E, Serra A, et al. Bipolar disorders and Wilson's disease. BMC Psychiatry. 2012;12:52.

6. Chien HF, Deguti MM, Cançado E, Azevedo RS, Scaff M, Barbosa ER. Neurological manifestations in Wilson's disease: report of 119 cases. Mov Disord. 2006;21(12):2192-96.

7. Kumar CP, Murthy P. Mania as a presenting symptom of Wilson's disease. Acta Neuropsychiatrica. 2006;18(1):47-9.

8. Jann MW. Diagnosis and Treatment of Bipolar Disorders in Adults: A Review of the Evidence on Pharmacologic Treatments. Am Health Drug Benefits. 2014;7(9):489-99. 